\title{
Die Erfassung von Lehransätzen von Lehrenden in der Weiterbildung - Entwicklung der Lehransatz-Skala
}

\author{
Caroline Bonnes $(\mathbb{D} \cdot$ Sabine Hochholdinger
}

Eingegangen: 20. Januar 2019 / Überarbeitet: 9. Juli 2020 / Angenommen: 12. Januar 2021 / Online publiziert: 27. Januar 2021

(C) Der/die Autor(en) 2021

Zusammenfassung Lehransätze sind ein bedeutsamer Faktor in der Gestaltung von Lehrsettings und für den Lernerfolg der Lernenden, wurden jedoch bisher noch nicht im Weiterbildungskontext untersucht. Bereits bestehende Instrumente aus der Hochschulforschung, wie das Approaches to Teaching Inventory, sind aufgrund der kontextspezifischen Items weniger geeignet für die Verwendung im Weiterbildungskontext. Für die zukünftige Erforschung von Lehransätzen in der Weiterbildung wurde daher ein Messinstrument mit 17 Items entwickelt, das transmissive (LAT) und konstruktivistische (LA-K) Lehransätze erfassen soll. Die Items wurden auf Basis einer qualitativen Vorstudie $(N=45)$ und einem Pretest entwickelt und in einer quantitativen Hauptstudie $(N=411)$ auf Reliabilität und Validität untersucht. Das Instrument wurde anhand einer explorativen Faktorenanalyse entwickelt und die Struktur der Skalen LA-T und LA-K durch exploratory structural equation modelling (ESEM) geprüft. Die Lehransatz-Skala bietet damit eine Basis für die weitere Erforschung von Lehransätzen im Weiterbildungskontext.

Schlüsselwörter Lehransätze · Lehrende in der Weiterbildung · Weiterbildung · Skalenentwicklung

Dr. C. Bonnes $(\bowtie) \cdot$ Prof. Dr. S. Hochholdinger

Lehrstuhl für Betriebspädagogik, Fachbereich Wirtschaftswissenschaften, Universität Konstanz, Universitätsstr. 10, 78457 Konstanz, Deutschland

E-Mail: caroline.bonnes@uni-konstanz.de

Prof. Dr. S. Hochholdinger

E-Mail: sabine.hochholdinger@uni-konstanz.de 


\title{
The measurement of approaches to teaching of trainers-Development of the Approaches-to-Teaching-Scale
}

\begin{abstract}
Approaches to teaching are an important factor for the design of teaching settings and for the learning outcome of learners. However, they have not been studied in the context of professional training. Existing instrument from higher education research, such as the Approaches to Teaching Inventory, were found to be not suitable for training research. Therefore, we developed a measurement instrument for future studies on approaches to teaching in professional training. It consists of 17 items and measures transmissive and constructivist approaches to teaching. Items were developed through a qualitative prestudy $(N=45)$, a pretest and a quantitative main study $(N=411)$ and tested for reliability and validity. The instrument was developed through exploratory factor analysis and the structure of the transmissive and constructivist subscales were confirmed via exploratory structural equation modelling (ESEM). The Approaches to Teaching Scale provides a basis for further research on approaches to teaching in professional training
\end{abstract}

Keywords Approaches to teaching · Trainers · Training · Scale development

\section{Einleitung}

Lehransätze beschreiben nach Trigwell et al. (1994) Intentionen bzw. Ziele, die eine Lehrperson im Lehrkontext verfolgt sowie Strategien bzw. Methoden, mit denen dieses Ziel erreicht werden soll. Die bisherige Forschung hat gezeigt, dass Lehransätze ein wichtiger Faktor für die Lehrenden-Lernenden-Interaktion sind und im Zusammenhang mit dem Lernerfolg der Lernenden stehen (z. B. Braun und Hannover 2008; Rosário et al. 2014; Trigwell und Prosser 2004). Lehransätze wurden bisher fast ausschließlich im Schul- und Hochschulkontext erforscht, obwohl davon auszugehen ist, dass das Konstrukt auch in der beruflichen Weiterbildung von Bedeutung ist. Bislang stehen jedoch keine passenden Instrumente zur Verfügung, die Lehransätze für die Erforschung im Weiterbildungskontext reliabel und valide operationalisieren. Das Ziel dieser Studie ist daher die Entwicklung eines Instrumentes zur Erfassung von Lehransätzen von Lehrenden in der beruflichen Weiterbildung. Im Folgenden werden wir zunächst das Konzept von Lehransätzen und die bisherige Forschung dazu vorstellen. Anschließend gehen wir auf die Messung von Lehransätzen ein und unterziehen das Konzept einer kritischen Betrachtung.

\subsection{Lehransätze als Komponente professioneller Kompetenz von Lehrenden in der Weiterbildung}

In der Forschung zur beruflichen Weiterbildung, insbesondere der arbeits- und organisationspsychologischen Trainingswirksamkeitsforschung, gibt es einen breiten Forschungskorpus an empirischen Arbeiten zu verschiedenen Einflussfaktoren auf den Erfolg von Weiterbildungen und Trainings (vgl. Aguinis und Kraiger 2009; Baldwin und Ford 1988; Blume et al. 2010; Burke und Hutchins 2007; Salas et al. 
Tab. 1 Lehransätze. (Trigwell et al. 1994, S. 78; Copyright 1994 Kluwer Academic Publishers, mit Genehmigung durch Springer)

\begin{tabular}{llll}
\hline Intention & $\begin{array}{l}\text { Strategy } \\
\text { Teacher-focused }\end{array}$ & Student/teacher interaction & Student-focused \\
\hline Information transmission & A & - & - \\
Concept acquisition & B & C & - \\
Conceptual development & - & - & D \\
Conceptual change & - & - & E \\
\hline
\end{tabular}

2012). Die Lehrenden als Einflussfaktor wurden dabei bisher weitgehend vernachlässigt (vgl. Baldwin et al. 2017; Hochholdinger und Leidig 2012). Die empirische Unterrichtsforschung zeigt jedoch, dass Lehrende und ihre professionelle Handlungskompetenz von hoher Bedeutung für den Lernerfolg der Lernenden sind (vgl. Baumert und Kunter 2006; König und Blömeke 2009). Eine Facette professioneller Handlungskompetenz von Lehrkräften stellen Lehransätze dar, die das Handeln der Lehrperson selbst und damit den Lernerfolg der Lernenden beeinflussen können (z. B. Braun und Hannover 2008; Trigwell and Prosser 2004).

Lehransätze wurden von Trigwell et al. (1994) als eine Kombination aus Intentionen und Strategien beschrieben, deren unterschiedliche Kombination in fünf verschiedenen Lehransätzen von A bis E resultiert (Tab. 1). Trigwell et al. (1994) beschreiben vier verschiedene Intentionen, die hierarchisch aufeinander aufbauen: Informationsweitergabe (information transmission), Erwerb von Konzepten (concept acqusition), Weiterentwicklung von Konzepten (conceptual development) und Veränderung von Konzepten bzw. Entwicklung neuer Konzepte (conceptual change). Die Strategien werden in drei Kategorien aufgeteilt: transmissiv/lehrendenorientiert (teacher-focused), Interaktion zwischen Lehrenden und Lernenden (student/teacher interaction) sowie konstruktivistisch/lernendenorientiert (student-focused).

Das Modell wurde im Rahmen einer qualitativen Interviewstudie mit 24 Hochschullehrenden der Chemie und Physik aufgestellt (Trigwell et al. 1994). Darauf aufbauend haben Trigwell und Prosser (2004) das Approaches to Teaching Inventory (ATI) entwickelt, das transmissive bzw. lehrendenorientierte Lehransätze und konstruktivistische bzw. lernendenorientierte Lehransätze erfassen soll. Bei einem transmissiven Ansatz steht der Inhalt im Vordergrund. Die Lehrperson hat die Rolle eines Experten und es werden vorwiegend transmissive Methoden (z. B. Weitergabe von Informationen) verwendet. Die Lernenden haben dabei eine passive Rolle und nehmen das ihnen vermittelte Wissen auf. Im Gegensatz dazu stehen bei einem konstruktivistischen Ansatz die Lernenden im Mittelpunkt des Lernprozesses. Die Lehrperson übernimmt die Rolle eines Lernbegleiters, nutzt eher konstruktivistische, kognitiv aktivierende Methoden und ermöglicht den Lernenden, den Lernprozess aktiv zu gestalten.

Während Kember (1997) diese beiden Orientierungen als die zwei entgegengesetzten Pole einer Skala beschreibt, konnte in der weiteren Forschung zu Lehransätzen gezeigt werden, dass sie nicht eindimensional, sondern zweidimensional sind (z.B. Braun und Hannover 2008; Lübeck 2010; Prosser und Trigwell 2006). Das bedeutet, dass die Ausprägung einer Lehrperson auf einer der beiden Dimensionen 
weitgehend unabhängig von der Ausprägung der anderen Dimension ist. Lehrende können also z.B. durchaus gleichzeitig hohe Ausprägungen auf der Dimension lehrendenorientiert als auch auf der Dimension lernendenorientiert aufweisen.

\subsection{Forschung zu Lehransätzen}

Bei Betrachtung der Forschung der letzten zwei Jahrzehnte wird deutlich, dass Lehransätze mit der Lehrgestaltung und dem Lernerfolg zusammenhängen. Lernende hatten bei konstruktivistischen Lehransätzen einen größeren subjektiven Kompetenzzuwachs (Braun und Hannover 2008) und zeigten eher ein tiefes Lernen (deep learning approach) (Rosário et al. 2014; Trigwell et al. 1999; Trigwell und Prosser 2004) im Gegensatz zum Oberflächenlernen (surface learning approach).

Die nach unserer Recherche einzige Studie, die Lehransätze im Bereich der beruflichen Weiterbildung untersucht hat, konnte zeigen, dass ein konstruktivistischer Lehransatz in Beziehung zu positiven emotionalen Reaktionen und Bewertungen der Teilnehmenden von Weiterbildungsmaßnahmen steht (Hochholdinger und Keller 2015). Darüber hinaus nutzen Lehrende in der Weiterbildung als auch Hochschullehrende mit einem konstruktivistischen Lehransatz mehr aktivierende Methoden (Braun und Hannover 2008; Hochholdinger und Keller 2015).

Bis auf die Studie von Hochholdinger und Keller (2015) wurden Lehransätze im Bereich der Erwachsenenbildung bzw. Weiterbildung bisher nicht weiter erforscht, obwohl zu vermuten ist, dass Lehransätze auch in diesem Bildungskontext von Bedeutung für die Gestaltung von Lehr-Lern-Situationen und für den Lernerfolg sind. Ähnlich wie in der Schule oder Hochschule werden Weiterbildungen von Lehrpersonen geplant und durchgeführt. Dabei ist wie für die ersten beiden Kontexte anzunehmen, dass die Lehrenden in der Weiterbildung unterschiedliche Intentionen und Strategien bei der Planung und Durchführung von Weiterbildungen aufweisen können. In Anbetracht der vorgestellten Studienlage ist daher davon auszugehen, dass Lehransätze auch im Zusammenhang mit der Gestaltung und dem Erfolg von Weiterbildungen stehen.

\subsection{Messung von Lehransätzen}

Um Lehransätze im Weiterbildungskontext messen zu können, wird ein valides und reliables Messinstrument benötigt. Das am häufigsten verwendete Instrument in den genannten Studien ist das Approaches to Teaching Inventory (ATI) von Trigwell und Prosser (2004). Seither wurde es in zahlreichen Studien in der Hochschulforschung (z. B. Fendler und Gläser-Zikuda 2013; Lindblom-Ylänne et al. 2006; Stes und Van Petegem 2014) und etwas seltener auch in der Schulforschung (z. B. Beausaert et al. 2013; Mak und Chik 2011) eingesetzt. Das ATI besteht aus 16 Items auf einer fünfstufigen Likert-Skala von trifft fast nie zu bis trifft fast immer zu und erfasst die beiden Subskalen transmission/teacher-focused (ITTF) (z.B. „In this subject my teaching focuses on the good presentation of information to students.") und conceptual change/student-focused (CCSF) (z.B. „I make available opportunities for students in this subject to discuss their changing understanding of the subject."). 
Bei der Betrachtung des Instrumentes wird der starke Bezug zum Hochschulkontext deutlich. Es verweist in mehreren Items auf die Vorbereitung der Studierenden auf die formale Prüfung durch die Lehrperson, z. B. „In this subject, I only provide the students with the information they will need to pass the formal assessments". In der beruflichen Weiterbildung sind formale Prüfungen oder Zertifizierungen weitaus seltener als im Hochschulkontext. Weiterhin beziehen sich zwei Items auf die Generierung von Mitschriften, was in der beruflichen Weiterbildung ebenfalls seltener vorkommt. Ebenso zeigt sich in der Beschreibung der einzelnen Ansätze (Trigwell et al. 1994) die konzeptuelle Verortung im Kontext von Hochschule und Fachdisziplin, z. B. durch Formulierungen wie ,transmitting to the students information about the discipline“" oder ,gain disciplinary knowledge“.

Weiterbildung im beruflichen Kontext bezieht sich auf geplante und systematische Handlungen, die die Aneignung von Wissen, Fähigkeiten und Einstellungen ermöglichen (Salas et al. 2012) und einen Bezug zum Arbeitsleben der Teilnehmenden aufweisen. Damit soll sie zum einen die Aneignung von Wissen und Verhalten unterstützen (Salas et al. 2012), zum anderen den Transfer des Gelernten in die Arbeitspraxis fördern. Trotz der Überschneidungen in den didaktischen Settings von Hochschule und Weiterbildung werden unterschiedliche Ziele und Intentionen verfolgt. In Hochschulveranstaltungen steht das Studieren eines spezifischen Faches im Vordergrund, in der Regel verbunden mit einer formalen Prüfung des Gelernten. Das zentrale Ziel von beruflichen Weiterbildungen hingegen ist die Übertragung des Gelernten in das Arbeitsleben. Damit wird insgesamt deutlich, dass die Konzeption von Lehransätzen nach Trigwell et al. (1994) und das daraus entstandene Instrument des ATI (Trigwell und Prosser 2004) nicht unmittelbar auf den Weiterbildungskontext übertragen werden können.

\subsection{Kritische Betrachtung des Konzeptes Lehransätze}

Sowohl das von Trigwell et al. (1994) entwickelte Konzept zu Lehransätzen als auch das darauf basierende ATI (Trigwell und Prosser 2004) wurden in der Vergangenheit kritisch betrachtet. Meyer und Eley (2006) beanstanden die Verallgemeinerung des Konzeptes auf die Lehransätze von Hochschullehrenden allgemein, obwohl das Konzept in einem spezifisch naturwissenschaftlichen Kontext entwickelt wurde. Weiterhin kritisieren sie Lehransatz D (Tab. 1), der nur auf einem einzigen Interview von insgesamt 24 basiert, und die zum Teil nicht nachvollziehbare Vorgehensweise bei der Entwicklung des ATI. Harshman und Stains (2017) evaluieren die Faktorenstruktur des ATI in bisherigen Studien als auch in einer eigenen Studie. Sie kommen zu dem Schluss, dass die von Trigwell und Prosser intendierte Faktorenstruktur (z. B. Prosser und Trigwell 2006; Trigwell und Prosser 2004) nicht durchgängig repliziert werden kann.

Die genannten Kritikpunkte und die zuvor aufgezeigten Unterschiede zwischen Hochschule und Weiterbildung legen nahe, das Konzept nicht ohne weitere Betrachtung weiterzuverwenden. Dennoch kommen wir zu dem Schluss, dass das Konzept der Lehransätze von Trigwell et al. (1994) eine geeignete theoretische Ausgangsbasis für ein Messinstrument in der beruflichen Weiterbildung darstellt. Die Stärke des Modells liegt unseres Erachtens nach darin, dass Lehransätze Intentionen und 
Strategien gemeinsam betrachten. Diese Kombination ermöglicht es zu untersuchen, welche Art des Lehrhandelns Lehrende in einer Weiterbildung zeigen und gleichzeitig zu betrachten, was die Lehrenden mit ihrem Handeln erreichen wollen. Damit sind Lehransätze stärker handlungsorientiert als beispielsweise Lehrüberzeugungen, ein verwandtes Konstrukt, das bisher vor allem in der empirischen Unterrichtsforschung untersucht wurde (vgl. Seifried 2009). Lehrüberzeugungen beziehen sich auf subjektive, implizite oder explizite Konzeptionen, z. B. im Rahmen von subjektiven Theorien zu Lehren und Lernen (Baumert und Kunter 2006). Kember (1997) stellt die These auf, dass Lehrkonzeptionen die Art des Lehransatzes beeinflussen und darüber der Lernansatz der Lernenden und damit einhergehend der Lernerfolg beeinflusst wird. In dieser postulierten Wirkungskette stehen Lehransätze in einem direkteren Zusammenhang mit dem Lernerfolg und sind auch in ihrer Konzeptualisierung näher am tatsächlichen Lehrhandeln.

\subsection{Ziel der Studie}

Die Sichtung der bisherigen empirischen Studien konnte zeigen, dass Lehransätze ein wichtiger Faktor für die Gestaltung und den Erfolg von Lehrsettings sind. Es ist daher zu vermuten, dass Lehransätze auch im beruflichen Weiterbildungskontext eine Rolle spielen und ihre Erforschung zu neuen Erkenntnissen für die Gestaltung von Weiterbildungsmaßnahmen führen würde. Das bisher am meisten verwendete Erhebungsinstrument für Lehransätze, das ATI, zeigt sich bei genauerer Betrachtung jedoch als ungeeignet für den Weiterbildungssektor. Unser Ziel ist es daher, ein reliables, valides und ökonomisches Messinstrument für Lehransätze in der beruflichen Weiterbildung zu entwickeln. Im Folgenden wird im Rahmen einer qualitativen Vorstudie (Bonnes und Hochholdinger 2020) und einer quantitativen Hauptstudie der Frage nachgegangen, wie Lehransätze im Weiterbildungskontext operationalisiert werden können und wie eine mögliche Faktorenstruktur sich ausgestaltet. Weiterhin wird eine erste Überprüfung der Validität vorgenommen.

\section{Methode}

Für die Entwicklung des Messinstrumentes wurden eine qualitative Vorstudie (Bonnes und Hochholdinger 2020), ein Pretest und eine quantitative Hauptstudie durchgeführt.

\subsection{Qualitative Vorstudie}

Das Ziel der qualitativen Vorstudie (Bonnes und Hochholdinger 2020) war zum einen, die Übertragbarkeit des Konzeptes von Lehransätzen auf den Weiterbildungskontext zu prüfen und es, wenn nötig, zu modifizieren. Zum anderen war es das Ziel, herauszufinden, wie sich die einzelnen Teilaspekte des Konzeptes genau im Weiterbildungskontext ausgestalten, um eine empirisch begründete Basis für die inhaltliche Struktur des Instrumentes und zur Formulierung von Items zu erhalten. 
Tab. 2 Kategoriensystem der qualitativen Vorstudie. (Bonnes und Hochholdinger 2020)

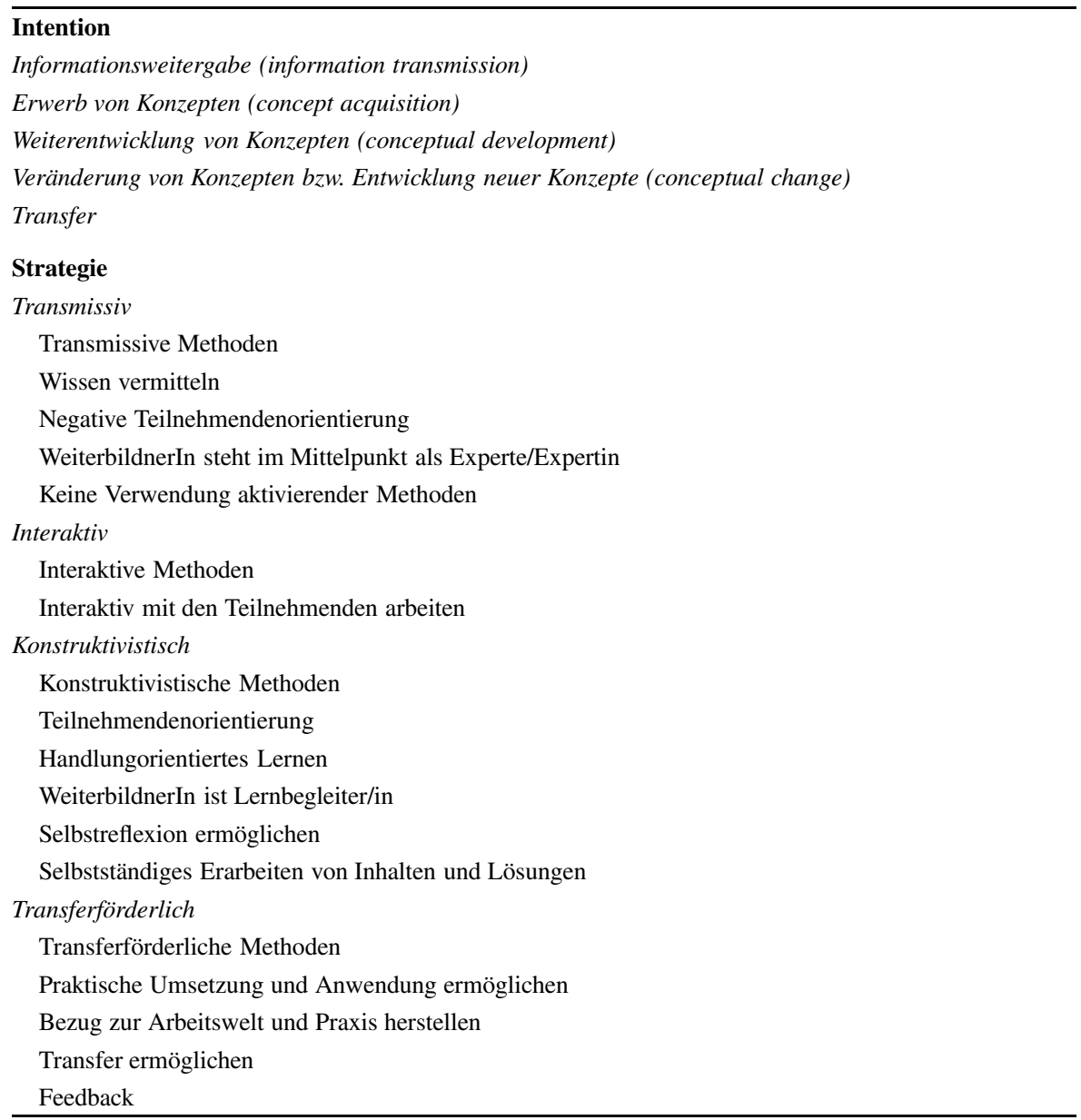

Anhand eines halbstrukturierten Interviewleitfadens wurden 23 Lehrpersonen aus der beruflichen Weiterbildung, 12 TeilnehmerInnen von Weiterbildungen und 10 PersonalentwicklerInnen, die Weiterbildungen planen und die Lehrpersonen auswählen, interviewt. Durch den Einbezug der Perspektive drei verschiedener Gruppen sollte ein umfassendes Bild von Lehransätzen im Weiterbildungskontext gewonnen werden.

Die Fragen bezogen sich vorwiegend darauf, was für die InterviewpartnerInnen eine gute/schlechte Weiterbildung sei und was für sie eine/n gute/n WeiterbildnerIn oder eine/n schlechte/n WeiterbildnerIn wäre. Als eher handlungsorientiertes Konstrukt zeigen sich Lehransätze vor allem in der Gestaltung einer Weiterbildung. Da Lehransätze jedoch auch ein Konstrukt sind, das in direkter Verbindung mit der Lehrperson steht, wurde ebenfalls direkt nach dem/der Weiterbildner/in gefragt. Dem Prinzip der Offenheit in der qualitativen Forschung folgend, haben wir uns 
dafür entschieden, diese sehr offenen Fragen zu stellen, anstatt z.B. direkte Fragen nach Weiterbildungszielen oder Methoden zu verwenden.

Die Interviews wurden mit Hilfe der qualitativen Inhaltsanalyse (vgl. Mayring 2015) analysiert. Es wurde eine Kombination aus deduktiver inhaltlicher Strukturierung und induktiver Zusammenfassung verwendet, um ggf. notwendige Erweiterungen oder Modifikationen des Lehransatzkonzeptes für den Weiterbildungskontext identifizieren zu können. Das Kategoriensystem wurde durchgehend während des Kodierprozesses im Forschungsteam diskutiert und gegebenenfalls modifiziert, bis sich aus dem Material keine weiteren Änderungen mehr für das Kategoriensystem ergaben. Das gesamte Material wurde anschließend von einer Person kodiert, mit $N=1404$ Kodierungen. Zur Bestimmung der Interkoderreliabilität wurde $50 \%$ des Materials von zwei weiteren Personen kodiert und Cohens Kappa (Cohen 1960) für die jeweiligen Kodiererpaare berechnet, das zwischen 0,77 und 0,84 lag. Das deduktiv entwickelte Kategoriensystem (Tab. 2) orientierte sich an Trigwell et al. (1994) (Tab. 1) und wurde im Rahmen der Analyse um die Intention-Kategorie Transfer und die Strategie-Kategorie transferförderliche Strategien ergänzt, da sich diese Thematik als ein neuer und eigenständiger inhaltlicher Aspekt in der Analyse zeigte. In der Vorstudie ließ sich nicht abschließend klären, ob die neuen Aspekte dem konstruktivistischen Lehransatz zuzuordnen sind, oder ob es sich dabei um unabhängige Konstrukte handelt. Dieser offenen Frage galt es im Rahmen der Instrumentenentwicklung nachzugehen.

Die Unterkategorien der Hauptkategorie Strategie ließen sich im Rahmen einer induktiven Analyse weiter differenzieren, um so eine genauere Beschreibung davon zu erhalten, welche Arten von Strategien z. B. einen konstruktivistischen Lehransatz ausmachen und damit eine gute Basis für die Entwicklung der Items bildeten.

\subsection{Pretest mit Experten}

Auf Grundlage der Ergebnisse der qualitativen Vorstudie und der Betrachtung der Items des ATI wurden 61 Items formuliert. Für jeden inhaltlichen Aspekt des Kategoriensystems gab es mehrere Alternativformulierungen, ausgenommen zur Rolle des Weiterbildenden, da allgemeine Aussagen zur Rolle nicht dem Formulierungsschema von Intentions- und Strategieitems entsprochen hätten. Für die Kategorie Feedback wurden ebenfalls keine Items formuliert, da diese Kategorie zum damaligen Zeitpunkt noch unter transferförderliche Methoden einsortiert war. Diese 61 Items wurden in einem Pretest mit 8 Lehrenden aus verschiedenen Weiterbildungskontexten mit der Think-Aloud-Technik (vgl. Bühner 2011) getestet, kritisch geprüft und ggf. modifiziert.

\subsection{Quantitative Hauptstudie}

\subsubsection{Stichprobenbeschreibung und Durchführung der Erhebung}

Die Daten wurden als Onlineumfrage erhoben. Der Link zur Studie wurde an Trainernetzwerke und an Weiterbildungsinstitutionen versendet und von dort aus weitergeleitet. Zusätzlich wurden Lehrpersonen aus dem Bereich der beruflichen Wei- 
terbildung über im Internet verfügbare Kontaktdaten angeschrieben. Am Ende der Datenerhebung hatten 581 Personen begonnen, den Fragebogen auszufüllen. Insgesamt bearbeiteten 411 Lehrende die Lehransatz-Skala, von denen 53,5\% $(n=220)$ männlich und 46,5\% $(n=191)$ weiblich waren. Das Alter der Lehrpersonen lag zwischen 24 und 78 Jahren $(M=50,1, S D=9,1)$. Für die Hälfte der Lehrenden $(51,1 \%)$ stellt die Tätigkeit als Lehrperson ihre Haupttätigkeit dar. Für 25,1 \% ist die Tätigkeit Teil des Spektrums der Haupttätigkeit, z. B. wenn eine Person als PersonalentwicklerIn arbeitet und zusätzlich interne Weiterbildungen durchführt, und 22,4\% gaben die Arbeit als Lehrperson als Nebentätigkeit an. Die Lehrenden hatten durchschnittlich 16 Jahre Berufserfahrung in der Lehrtätigkeit $(\mathrm{SD}=8,6)$. Allgemein kann zwischen wissensorientierten Weiterbildungen (z. B. zu Fachthemen wie Recht oder EDV) und verhaltensorientierten Weiterbildungen (z. B. zu Kommunikation oder Führung) unterschieden werden (Schaper und Sonntag 2007; Sonntag und Stegmaier 2007). Von den Lehrenden bieten 43,1\% vorwiegend verhaltensorientierte und 28,7\% vorwiegend wissensorientierte Veranstaltungen an, und 28,2\% gaben an, beide Arten von Veranstaltungen anzubieten. Die durchschnittliche Anzahl der Teilnehmenden bei der Weiterbildung, auf welche sich die Beantwortung der Lehransatz-Skala bezog, lag zwischen 3 und 150 Teilnehmenden $(M=14,8, S D=11,0)$ und die durchschnittliche Dauer der Weiterbildungen zwischen 1,5 und 480h $(\mathrm{M}=30,2, \mathrm{SD}=43,9)$.

\subsubsection{Instrumente}

Lehransätze Da Lehransätze kontext- bzw. veranstaltungsspezifisch sein können (Lindblom-Ylänne et al. 2006), beginnt der Fragebogen mit der Instruktion, an eine Veranstaltung zu denken, die die Lehrperson öfter hält, oder die am ehesten repräsentativ für ihre ansonsten durchgeführten Veranstaltungen ist. Dabei soll die letzte Durchführung möglichst nicht länger als ein Jahr zurückliegen. Die Fragen werden dann in Bezug auf diese spezifische Veranstaltung beantwortet.

Die Intention-Items wurden auf einer sechsstufigen Likert-Skala von trifft überhaupt nicht zu (1) bis trifft voll und ganz zu (6) und die Strategie-Items auf einer sechsstufigen Likert-Skala von nie (1) bis in sehr hohem Maße (6) beantwortet. Bei allen Items gab es die zusätzliche Antwortmöglichkeit kann ich nicht beurteilen als eine weitere Möglichkeit, kritische oder nicht leicht $\mathrm{zu}$ beantwortende Items zu identifizieren. Die entsprechende Kombination aus transmissiven bzw. konstruktivistischen Intention- und Strategieitems ergeben die beiden Subskalen transmissiver Lehransatz (LA-T) und konstruktivistischer Lehransatz (LA-K).

Angaben zur Veranstaltung Um mehr über die Veranstaltung zu erfahren, auf die sich die Lehrenden bei der Beantwortung der Lehransatzskala bezogen, wurden zusätzlich die Anzahl der Teilnehmenden und die Dauer der Veranstaltung in Stunden erfragt. Weiterhin wurde den Lehrenden eine Liste mit verschiedenen Methoden vorgelegt, mit der Bitte um Einschätzung, in welchem Maße sie diese Methoden in der Veranstaltung verwendet haben, von nie (1) bis in sehr hohem Maße (6). 


\subsubsection{Vorannahmen zur Skalenstruktur und Überprüfung der Kriteriumsvalidität}

Die folgenden Vorannahmen zur Skalenstruktur lassen sich aus der bisherigen Literatur ableiten. Lehrkonzeptionen - als ein den Lehransätzen übergeordnetes Konstrukt - werden von Kember (1997) als dichotome Variable mit den beiden Ausprägungen lehrenden- und lernendenorientiert gesehen. Ebenso basiert das ATI (Trigwell und Prosser 2004) auf den zwei entsprechenden Subskalen. Daher wird für die Messung von Lehransätzen von Lehrenden in der Weiterbildung ebenfalls ein Messmodell mit zwei Subskalen (transmissiver Lehransatz und konstruktivistischer Lehransatz) angenommen. Obwohl die theoretischen Modelle hinter den gemessenen Konstrukten zum Teil differenzierter sind, hat sich beispielsweise beim ATI gezeigt, dass die einzelnen Zwischenkategorien sich nicht als selbstständige Skala abbilden lassen (Prosser und Trigwell 2006; Trigwell und Prosser 2004). Sowohl die konstruktivistische als auch die transmissive Subskala sollen sich - ebenfalls in Anlehnung an das ATI - aus einer Kombination von Items zu Intentionen und Strategien bilden.

In bisherigen Studien hat sich gezeigt, dass die beiden Subskalen weitgehend unabhängig voneinander sind bzw. eine geringe bis mittlere negative Korrelation aufzeigen (z. B. Braun und Hannover 2008: $r=0$,05; Lübeck 2010: $r=-0,23$; Trigwell und Prosser 2004: $r=-0,30$ ). Daher wird für die hier entwickelte LehransatzSkala ebenfalls erwartet, dass die beiden Subskalen eine geringe bis mittlere negative Korrelation aufweisen.

Zusammenfassend lassen sich damit drei Teilhypothesen zur Skalenstruktur formulieren: (1a) Die Lehransatz-Skala besteht aus zwei Subskalen, die jeweils einen transmissiven (LA-T) oder konstruktivistischen (LA-K) Lehransatz beschreiben. (1b) Die Subskalen bestehen jeweils aus Items, die Intentionen und Strategien beschreiben. (1c) Die beiden Subskalen weisen eine geringe bis mittlere negative Korrelation auf.

Für eine erste Überprüfung der Kriteriumsvalidität wird der Zusammenhang der beiden Subskalen mit den angegebenen verwendeten Methoden untersucht. Es ist davon auszugehen, dass ein transmissiver oder konstruktivistischer Lehransatz mit den verwendeten Methoden im Zusammenhang steht (Braun und Hannover 2008; Hochholdinger und Keller 2015). Es ist zu erwarten, dass (2a) hohe Werte auf der LA-T Skala mit hohen Werten bei der Verwendung transmissiver Methoden (z. B. Vortrag/Präsentieren von Inhalten durch die Lehrperson) und (2b) mit niedrigeren Werten bei der Verwendung konstruktivistischer Methoden (z. B. Diskussion in Kleingruppen und Rollenspiele) einhergehen. Für Lehrende mit hohen Werten auf der Skala LA-K wären die entgegengesetzten Zusammenhänge zu erwarten, d.h. dass $(3 a)$ hohe Werte auf der Skala LA-K im Zusammenhang mit niedrigeren Werten bei der Verwendung transmissiver Methoden und (3b) mit höheren Werten bei der Verwendung konstruktivistischer Methoden stehen. 


\section{Ergebnisse}

Ziel ist die Entwicklung eines reliablen, validen und ökonomischen Messinstrumentes. Für die Entwicklung der Skala und die Überprüfung der Faktorenstruktur wurde der Datensatz $(N=411)$ zunächst anhand einer Zufallsstichprobenziehung in zwei Datensätze für eine Kreuzvalidierung aufgeteilt. Stichprobe $1(N=200)$ wird für die Entwicklung der Skala verwendet und Stichprobe $2(N=211)$ für die Überprüfung der Faktorenstruktur.

\subsection{Entwicklung des Instruments}

Im Rahmen des ersten Selektierungsprozesses wurden die Kennwerte (Mittelwert, Streuung, Verteilung) und die Formulierung aller Items kritisch geprüft. Insgesamt wurden 39 der 61 Items gelöscht. Davon wurden 12 Items aufgrund sehr hoher Mittelwerte $(M \geq 5,3)$ herausgenommen. Darunter befanden sich vorwiegend Items der Kategorie Intention - Transfer und der Kategorie Intention - concept acquisition. Weitere 16 Items wiesen eine redundante Formulierung zu anderen Items auf. Alle Formulierungen wurden ebenfalls noch einmal kritisch daraufhin überprüft, ob sie eindeutig eine Intention bzw. Strategie erfassen, wodurch weitere 4 Items gestrichen wurden. Items, die bei nochmaliger Betrachtung missverständlich oder nicht eindeutig formuliert waren (7 Items), wurden ebenfalls entfernt.

Tab. 3 Faktorladungen EFA und ESEM

\begin{tabular}{lllll}
\hline & Faktorladungen EFA & & \multicolumn{2}{l}{ Faktorladungen ESEM } \\
& LA-T & LA-K & LA-T & LA-K \\
\hline ST1 & $\mathbf{0 , 4 4}$ & 0,00 & $\mathbf{0 , 4 3}$ & $-0,16$ \\
ST2 & $\mathbf{0 , 5 5}$ & $-0,05$ & $\mathbf{0 , 4 7}$ & $-0,20$ \\
ST3 & $\mathbf{0 , 5 0}$ & $-0,12$ & $\mathbf{0 , 4 2}$ & $-0,21$ \\
ST4 & $\mathbf{0 , 7 2}$ & $-0,20$ & $\mathbf{0 , 5 7}$ & $-0,36$ \\
IT1 & $\mathbf{0 , 8 9}$ & 0,17 & $\mathbf{0 , 8 5}$ & $-0,01$ \\
IT2 & $\mathbf{0 , 8 0}$ & 0,11 & $\mathbf{0 , 7 1}$ & $-0,01$ \\
IT3 & $\mathbf{0 , 7 6}$ & 0,05 & $\mathbf{0 , 8 4}$ & 0,08 \\
IT4 & $\mathbf{0 , 6 1}$ & 0,02 & $\mathbf{0 , 7 8}$ & 0,12 \\
SK1 & 0,12 & $\mathbf{0 , 4 1}$ & 0,19 & $\mathbf{0 , 5 6}$ \\
SK2 & 0,04 & $\mathbf{0 , 4 8}$ & $-0,02$ & $\mathbf{0 , 6 1}$ \\
SK3 & $-0,07$ & $\mathbf{0 , 6 9}$ & $-0,01$ & $\mathbf{0 , 7 0}$ \\
SK4 & $-0,05$ & $\mathbf{0 , 3 9}$ & $-0,09$ & $\mathbf{0 , 4 9}$ \\
SK5 & 0,05 & $\mathbf{0 , 3 6}$ & 0,11 & $\mathbf{0 , 4 2}$ \\
IK1 & $-0,07$ & $\mathbf{0 , 6 9}$ & $-0,06$ & $\mathbf{0 , 6 4}$ \\
IK2 & 0,05 & $\mathbf{0 , 7 6}$ & 0,10 & $\mathbf{0 , 7 8}$ \\
IK3 & $-0,12$ & $\mathbf{0 , 7 2}$ & $-0,10$ & $\mathbf{0 , 6 6}$ \\
IK4 & 0,07 & $\mathbf{0 , 7 0}$ & 0,08 & $\mathbf{0 , 6 2}$ \\
\hline
\end{tabular}

$N_{\text {EFA }}=177, \mathrm{~N}_{\mathrm{ESEM}}=211$

EFA Explorative Faktorenanalyse, ESEM exploratory structural equation modelling, ST transmissive Strategie, $S K$ konstruktivistische Strategie, $I T$ transmissive Intention, $I K$ konstruktivistische Intention

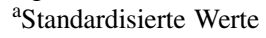


Tab. 4 Itemkennwerte

\begin{tabular}{|c|c|c|c|c|c|}
\hline & & $M$ & $S D$ & $R_{i t}$ & $P_{i}$ \\
\hline \multicolumn{6}{|c|}{$L A-T$} \\
\hline ST1 & $\begin{array}{l}\text { Ich bin aktiv gewesen und die Teilnehmenden } \\
\text { haben zugehört }\end{array}$ & 3,45 & 1,29 & 0,45 & 0,49 \\
\hline ST2 & Ich habe den Teilnehmenden Inhalte präsentiert & 3,98 & 1,34 & 0,55 & 0,60 \\
\hline ST3 & $\begin{array}{l}\text { Ich habe Inhalte durch Vorträge oder Präsenta- } \\
\text { tionen vermittelt }\end{array}$ & 3,74 & 1,40 & 0,55 & 0,55 \\
\hline ST4 & $\begin{array}{l}\text { Der Schwerpunkt der Veranstaltung lag auf der } \\
\text { Vermittlung von Wissen und Informationen }\end{array}$ & 3,95 & 1,54 & 0,71 & 0,59 \\
\hline IT1 & $\begin{array}{l}\text { Die Teilnehmenden sollten Wissen/ } \\
\text { Informationen vermittelt bekommen }\end{array}$ & 4,56 & 1,33 & 0,72 & 0,71 \\
\hline IT2 & $\begin{array}{l}\text { Die Teilnehmenden sollten fachliche Inhalte } \\
\text { vermittelt bekommen, um eine Wissensbasis zu } \\
\text { diesem Thema aufzubauen }\end{array}$ & 4,49 & 1,39 & 0,63 & 0,70 \\
\hline IT3 & $\begin{array}{l}\text { Die Teilnehmenden sollten Daten und Fakten } \\
\text { zum Thema vermittelt bekommen }\end{array}$ & 3,89 & 1,46 & 0,68 & 0,58 \\
\hline IT4 & $\begin{array}{l}\text { Die Teilnehmenden sollten Inhalte lernen, die } \\
\text { auch in (Fach-)Büchern zum Thema stehen }\end{array}$ & 3,82 & 1,50 & 0,54 & 0,56 \\
\hline \multicolumn{6}{|c|}{$L A-K$} \\
\hline SK1 & $\begin{array}{l}\text { Ich habe flexibel auf den Bedarf der Teilneh- } \\
\text { menden reagiert }\end{array}$ & 5,02 & 0,98 & 0,36 & 0,80 \\
\hline SK2 & $\begin{array}{l}\text { Die Teilnehmenden konnten über die Inhalte } \\
\text { miteinander diskutieren }\end{array}$ & 4,79 & 1,34 & 0,45 & 0,76 \\
\hline SK3 & $\begin{array}{l}\text { Die Teilnehmenden haben die Möglichkeit zur } \\
\text { Selbstreflexion erhalten }\end{array}$ & 4,91 & 1,16 & 0,66 & 0,78 \\
\hline SK4 & $\begin{array}{l}\text { Die Teilnehmenden haben selbständig Lösungs- } \\
\text { vorschläge für Aufgaben oder Problemstellun- } \\
\text { gen erarbeitet }\end{array}$ & 4,63 & 1,18 & 0,37 & 0,73 \\
\hline SK5 & $\begin{array}{l}\text { Ich habe den Teilnehmenden die Möglichkeit } \\
\text { gegeben, das Gelernte in die Praxis zu übertra- } \\
\text { gen }\end{array}$ & 4,83 & 1,14 & 0,32 & 0,77 \\
\hline IK1 & $\begin{array}{l}\text { Die Teilnehmenden sollten sich persönlich wei- } \\
\text { terentwickeln }\end{array}$ & 4,98 & 1,35 & 0,60 & 0,80 \\
\hline IK2 & $\begin{array}{l}\text { Die Teilnehmenden sollten ihre Sicht auf das } \\
\text { Thema hinterfragen }\end{array}$ & 4,69 & 1,42 & 0,63 & 0,74 \\
\hline IK3 & $\begin{array}{l}\text { Die Teilnehmenden sollten Veränderungsimpul- } \\
\text { se bekommen }\end{array}$ & 4,93 & 1,41 & 0,65 & 0,79 \\
\hline IK4 & $\begin{array}{l}\text { Ich wollte, dass die Veranstaltung Transfer er- } \\
\text { möglicht }\end{array}$ & 5,19 & 1,06 & 0,61 & 0,84 \\
\hline
\end{tabular}

$N=200$

$r_{i t}$ Item-Trennschärfe, $P_{i}$ Itemschwierigkeit, $L A-T$ transmissiver Lehransatz, $L A-K$ konstruktivistischer Lehransatz, $S T$ transmissive Strategie, $S K$ konstruktivistische Strategie, $I T$ transmissive Intention, $I K$ konstruktivistische Intention

Die verbleibenden 22 Items wurden anschließend daraufhin untersucht, welche Kategorien aus der qualitativen Studie noch repräsentiert wurden. Es zeigte sich, dass die Kategorie Intention - concept acquisition aufgrund der hohen Mittelwerte nicht mehr vertreten war. Daher wurden auch die 3 Items, die sich auf die Strate- 
giekategorie Interaktion bezogen, aus der Skala herausgenommen, da Lehransatz C (Tab. 1) damit nicht mehr abgebildet werden konnte.

Für die Entwicklung der Skala wurde mit Stichprobe 1 eine explorative Faktorenanalyse (EFA) mit SPSS 25 durchgeführt. Die Angemessenheit der Stichprobe für eine explorative Faktorenanalyse bestätigen ein Kaiser-Meyer-Olkin-Kriterium von 0,81 und der Wert von 1360,20 $(p<0,001)$ für den Bartlett-Test auf Sphärizität. Die Überprüfung der Skalenstruktur wurde mit einer Hauptachsenanalyse durchgeführt. Aufgrund der theoretischen und empirischen Ausgangslage ist zu erwarten, dass die Faktoren miteinander korrelieren, so dass eine oblique Rotation (Promax) verwendet wurde. Zur Bestimmung der Anzahl der Faktoren wurde der Minimum-Average-Partial-Test (MAP-Test) nach Velicer et al. (2000) angewendet. Das Ergebnis des MAPTests empfiehlt die Vorgabe von drei Faktoren. Items, die transmissive Strategien und Intention erfassen, laden auf den ersten Faktor. Items, die konstruktivistische Strategien und Intention erfassen, laden auf den zweiten und dritten Faktor, jedoch ohne eine klare inhaltliche Zuordnung und mit drei Items, die auf beiden Faktoren laden. Daher werden im zweiten Schritt zwei Faktoren vorgegeben. Das Item, das negative Teilnehmendenorientierung erfasst, sowie ein weiteres konstruktivistisches Strategieitem laden unter 0,3 und werden daher entfernt. Die finale Version zeigt mit 17 Items eine klare Faktorenlösung aus zwei Faktoren, die einen transmissiven Lehransatz (LA-T) und einen konstruktivistischen Lehransatz (LA-K) repräsentieren und zusammen 47,2\% der Varianz aufklären (Tab. 3). Die Skala LA-T besteht aus acht Items, von denen vier transmissive Strategien und vier transmissive Intentionen erfassen. Die Skala LA-K bildet sich aus neun Items, von denen fünf Items konstruktivistische Strategien und vier Items konstruktivistische Intentionen erfassen.

Tab. 4 gibt eine Übersicht der Itemkennwerte für die Skala LA-T und die Skala LA-K. Die Trennschärfen der Items der Skala LA-T liegen zwischen 0,45 und 0,72 und weisen damit eine gute Streuung im empfohlenen Bereich zwischen 0,40 und 0,70 auf (Kelava und Moosbrugger 2012). Die Itemschwierigkeit liegt vorwiegend im mittleren bis niedrigeren Bereich von 0,49 bis 0,71. Die Trennschärfen der Items der Skala LA-K liegen zwischen 0,32 und 0,66 und sind damit etwas niedriger als bei der Skala LA-T. Die Itemschwierigkeit liegt mit 0,73 bis 0,84 im eher niedrigen Bereich.

\section{2 Überprüfung der Faktorenstruktur}

Für die Prüfung der Faktorenstruktur anhand von Stichprobe 2 wird das Verfahren des exploratory structural equation modeling (ESEM, Asparouhov und Muthén 2009) mit Mplus 7 angewendet, das EFA und konfirmatorische Faktorenanalyse (CFA) kombiniert. Marsh et al. (2014) argumentieren, dass ESEM die jeweiligen Stärken von EFA und CFA miteinander kombiniert, wobei sie ESEM dennoch als vorwiegend konfirmatorischen Ansatz sehen. ESEM eignet sich insbesondere für mehrdimensionale Konstrukte, da - anders als bei der CFA - Querladungen zwischen den Faktoren zugelassen und damit die Faktorenstruktur besser abgebildet werden kann (Marsh et al. 2010, 2014). 
Tab. 5 Modell-Fit des Basismodells und unter Hinzunahme weiterer Modellspezifikationen

\begin{tabular}{|c|c|c|c|c|c|c|c|c|c|c|}
\hline $\begin{array}{l}\text { Korrelation } \\
\text { Residuen }\end{array}$ & $\begin{array}{l}\text { Inhaltlicher } \\
\text { Bezug }\end{array}$ & M.I. & $\chi^{2}$ & $d f$ & $p$ & $\begin{array}{l}\text { CMIN/ } \\
\text { DF }\end{array}$ & CFI & TLI & RMSEA & SRMR \\
\hline - & - & & 266,117 & 103 & $<0,001$ & 2,58 & 0,87 & 0,82 & 0,09 & 0,06 \\
\hline $\begin{array}{l}\text { Items } \\
\text { IK1 \& IK3 }\end{array}$ & $\begin{array}{l}\text { Veränderung/ } \\
\text { Weiter- } \\
\text { entwicklung }\end{array}$ & 28,1 & 236,644 & 102 & $<0,001$ & 2,32 & 0,89 & 0,85 & 0,08 & 0,06 \\
\hline $\begin{array}{l}\text { Items } \\
\text { ST2 \& ST3 }\end{array}$ & $\begin{array}{l}\text { Inhalte prä- } \\
\text { sentieren }\end{array}$ & 24,4 & 211,560 & 101 & $<0,001$ & 2,09 & 0,91 & 0,88 & 0,07 & 0,05 \\
\hline $\begin{array}{l}\text { Items } \\
\text { SK4 \& SK5 }\end{array}$ & $\begin{array}{l}\text { Handlungs- } \\
\text { orientierung }\end{array}$ & 18,2 & 193,430 & 100 & $<0,001$ & 1,93 & 0,92 & 0,90 & 0,07 & 0,05 \\
\hline
\end{tabular}

M.I. Modification Index

Da die Daten nicht normalverteilt sind, wird der MLR-Schätzer (Maximum-Likelihood Robust) verwendet. Für die Faktorrotation wird wie bei der EFA ein obliques Verfahren (Geomin) angewandt. Für die Überprüfung des Modell-Fits wird eine Kombination aus $\chi^{2}$, CFI, TLI, RMSEA, SRMR verwendet (Kline 2016; Wang und Wang 2012). Zur Beurteilung des Modell-Fits wurden in der Vergangenheit unterschiedliche Schwellenwerte empfohlen. Hu und Bentler (1999) empfehlen die folgenden Schwellenwerte für einen guten Modell-Fit: CFI $>0,95$, RMSEA $<0,05$ und SRMR $<0,08$. Hu und Bentler (1999) sehen diese Werte jedoch nicht als absolut an, sondern als Richtwerte (Kline 2016). Little (2013) empfiehlt verschiedene Abstufungen für die Interpretation des Modell-Fits. Ein CFI von >0,90 indiziert einen akzeptablen Fit, während mit $>0,95$ ein guter Fit erreicht ist. Für RMSEA und SRMR bewertet er $<0,08$ als akzeptablen Fit und $<0,05$ als guten Fit. Für die $\chi^{2}-$ Statistik wird zusätzlich das Verhältnis zwischen $\chi^{2}$ und Freiheitsgraden (CMIN/DF) berichtet, das $<3$ sein sollte (Schermelleh-Engel et al. 2003).

Das Modell weist zunächst einen nicht akzeptablen Modell-Fit auf $\left(\chi^{2}=266,117\right.$, $d f=103, p<0,001, \mathrm{CMIN} / \mathrm{DF}=2,58, \mathrm{CFI}=0,87, \mathrm{TLI}=0,82, \mathrm{RMSEA}=0,09, p=0,00$, $90 \%$ C.I. $[0,07,0,10]$, SRMR=0,06). Die Modification Indices verweisen auf relevante Zusammenhänge zwischen den Residuen der Items. Modellspezifikationen sollten nur in Betracht gezogen werden, wenn sie auch inhaltlich begründbar sind (Wang und Wang 2012). Da die drei größten Modification Indices auf Items verweisen, die jeweils ähnliche inhaltliche Aspekte erfassen, sehen wir eine Modellspezifikation als inhaltlich sinnvoll an. Die erste Spezifikation bezieht sich auf Item IK1 (Die Teilnehmenden sollten sich persönlich weiterentwickeln) und IK3 (Die Teilnehmenden sollten Veränderungsimpulse bekommen). Beide beinhalten den Aspekt der Veränderung bzw. Weiterentwicklung. Item ST2 (Ich habe den Teilnehmenden Inhalte präsentiert) und ST3 (Ich habe Inhalte durch Vorträge oder Präsentationen vermittelt) verweisen beide darauf, Inhalte zu präsentieren. Item SK4 (Die Teilnehmenden haben selbständig Lösungsvorschläge für Aufgaben oder Problemstellungen erarbeitet) und SK5 (Ich habe den Teilnehmenden die Möglichkeit gegeben, das Gelernte in die Praxis zu übertragen) zeigen jeweils einen starken Bezug zur Handlungsorientierung. Eine Übersicht der Modifikationen, der jeweiligen inhaltlichen Aspekte und der veränderten Fit-Statistiken findet sich in Tab. 5. Nachdem die Korrelationen zwischen den Items im Modell schrittweise spezifiziert werden, weist das 
Modell einen akzeptablen bis guten Modell-Fit auf $\left(\chi^{2}=193,430, d f=100, p<0,001\right.$, $\mathrm{CMIN} / \mathrm{DF}=1,93, \mathrm{CFI}=0,92, \mathrm{TLI}=0,90, \mathrm{RMSEA}=0,07, p=0,03,90 \%$ C.I. $[0,05$, $0,08], \mathrm{SRMR}=0,05)$.

\subsection{Interne Konsistenz, Skalenstruktur und Kriteriumsvalidität}

Die folgenden Berechnungen wurden mit Stichprobe $2(N=211)$ durchgeführt. Die Reliabilität der LA-T sowie die der LA-K Skala zeigen mit einem Cronbachs Alpha von 0,88 bzw. 0,85 eine gute interne Konsistenz. Da Cronbachs Alpha u.a. von tauÄquivalenz ausgeht und kein Konfidenzintervall ausgibt (Dunn et al. 2014), wurde zusätzlich McDonalds Omega mit dem R-Paket von Peters (2018) berechnet. Omega liegt für LA-T bei $0,88(95 \%$ CI $[0,86,0,91])$ und für LA-K bei 0,85 (95\% CI [0,81, $0,88]$ ) und zeigt damit eine ähnlich gute interne Konsistenz an.

In Bezug auf die Hypothesen zur Skalenstruktur können alle drei Teilhypothesen angenommen werden. Die Lehransatz-Skala besteht (1a) aus zwei Subskalen, die jeweils einen transmissiven oder konstruktivistischen Lehransatz abbilden und (1b) jeweils aus Items zusammengesetzt sind, die Intentionen und Strategien beschreiben. Weiterhin (1c) stehen die Skalen LA-T und LA-K miteinander in einem mittleren negativen Zusammenhang $(r=-0,42, p<0,01)$.

Die Kriteriumsvalidität wird über die Angabe der verwendeten Methoden überprüft. Für die Skala LA-T zeigt sich (2a) ein hoher positiver Zusammenhang mit der transmissiven Methode Vortrag/Präsentieren von Inhalten durch die Lehrperson $(r=0,73, p<0,01)$ und $(2 b)$ ein negativer Zusammenhang mit den konstruktivistischen Methoden Diskussion in Kleingruppen $(r=-0,39, p<0,01)$ und Rollenspiel $(r=-0,41, p<0,01)$. Die Skala LA-K $(3 a)$ korrelierte negativ mit der transmissiven Methode $(r=-0,43, p<0,01)$ und $(3 b)$ positiv mit den beiden konstruktivistischen Methoden $(r=0,65, p<0,01$ und $r=0,55, p<0,01)$. Damit können alle Hypothesen zur Kriteriumsvalidität $(2 a, 2 b, 3 a, 3 b)$ angenommen werden.

\section{Diskussion}

Ziel unserer Studie war die Entwicklung eines Messinstrumentes zur Erfassung von transmissiven und konstruktivistischen Lehransätzen in der Weiterbildung. Auf Basis einer qualitativen Vorstudie und eines Pretests wurden 61 Items entwickelt, die im Rahmen einer Onlinestudie mit 411 Lehrenden in der Weiterbildung überprüft wurden. Die erste Itemselektion ergab aufgrund statistischer und inhaltlicher Prüfung eine Version mit 22 Items. Durch eine explorative Faktorenanalyse wurde eine Version mit 17 Items entwickelt, die auf zwei Faktoren laden, welche einen transmissiven Lehransatz (LA-T) mit acht Items und einen konstruktivistischen Lehransatz (LA-K) mit neun Items beschreiben. Die Faktorenstruktur der Skala wurde anschließend mit dem ESEM-Verfahren überprüft.

Das ursprüngliche Ziel war es, das Konstrukt möglichst so abzubilden, wie es sich in der qualitativen Vorstudie mit den verschiedenen inhaltlichen Aspekten gezeigt hat, um eine möglichst hohe Inhaltsvalidität $\mathrm{zu}$ erhalten. Jedoch zeigte sich bereits in der ersten Itemselektion, dass alle Items zur Intention concept acquisiti- 
on sehr hohe Mittelwerte aufwiesen und damit nicht für die Verwendung in einer Skala geeignet sind. Concept acquisition bezieht sich darauf, dass die Lehrperson das Wissen nicht nur vermitteln möchte, sondern ebenfalls erreichen möchte, dass die Teilnehmenden die vermittelten Inhalte verstehen und miteinander in Beziehung setzen können. Diese Intention scheint für den Großteil der Lehrpersonen einen sehr hohen Stellenwert zu haben und differenziert damit nicht mehr ausreichend. Lehransatz C konnte somit nicht mehr abgebildet werden. Auch bei der Entwicklung und Prüfung des ATI (Prosser und Trigwell 2006; Trigwell und Prosser 2004) konnte der mittlere Lehransatz nicht abgebildet werden. Die vier Intentionskategorien und die drei Strategiekategorien im Modell von Trigwell et al. (1994) stellen kontinuierliche Ausprägungen mit jeweils zwei Extremen dar. Es erscheint somit sinnvoll, Lehransätze mit zwei Skalen zu konzeptualisieren, die jeweils die extremeren Ausprägungen abbilden, um somit die Unterschiede in den Lehransätzen von Lehrpersonen zu erfassen. Auch in unserer EFA zeigte sich erst in der Version mit zwei Faktoren eine inhaltlich klare Faktorenstruktur.

Der Aspekt negative Teilnehmendenorientierung konnte aufgrund zu geringer Ladung auf dem transmissiven Faktor nicht beibehalten werden. Dies könnte entweder bedeuten, dass negative Teilnehmendenorientierung kein Teilaspekt eines transmissiven Lehransatzes ist, oder dass der Aspekt nicht gut erfasst wurde. Bereits in der Konstruktion der Items für diesen Aspekt zeigte sich die Schwierigkeit, negative Teilnehmendenorientierung in einem Item zu erfassen, ohne sozial erwünschte Antworten zu begünstigen. Dennoch wies das möglichst neutral formulierte Item „Das Erreichen des zuvor gesetzten Lernziels hatte höhere Priorität, als die individuellen Bedarfe der Teilnehmenden“"immer noch einen im Vergleich zu den anderen transmissiven Items sehr niedrigen Mittelwert von 2,9 auf. Anhand unserer Daten lässt sich die Frage nach der Zugehörigkeit zum transmissiven Lehransatz nicht abschließend klären und sollte in weiteren Studien untersucht werden.

Der Aspekt Teilnehmendenorientierung zeigte auf dem konstruktivistischen Faktor hingegen eine niedrige, aber noch akzeptable Ladung. Das Prinzip der Teilnehmendenorientierung betont, dass die „Voraussetzungen, Erwartungen, Lernstile und kognitiven Strukturen der Lernenden“ (Schrader 2010, S. 284) berücksichtigt werden müssen und kann damit als Voraussetzung und Bestandteil eines konstruktivistischen Lehransatzes gesehen werden. Dennoch sollte in Anbetracht der Ergebnisse für die negative Teilnehmendenorientierung auch dieser Aspekt in zukünftigen Studien vertieft betrachtet werden.

Der in der qualitativen Vorstudie zusätzlich identifizierte Aspekt des Transfers konnte als konstruktivistische Intention und im Rahmen der konstruktivistischen Strategieitems abgebildet werden. Für die Intentionsitems zeigte sich jedoch ebenfalls das Problem sehr hoher Mittelwerte. Dies spricht dafür, dass die Lehrpersonen in unserer Stichprobe eine sehr stark ausgeprägte Intention zeigen, dass die Teilnehmenden das Gelernte in ihre Praxis übertragen können. Das verbliebene Transfer-Intentionsitem zeigt eine ähnlich gute Ladung wie die anderen konstruktivistischen Intentionsitems. Dies spricht dafür, dass im Weiterbildungskontext Transfer ein Bestandteil einer konstruktivistischen Intention ist. Die Strategieitems mit Transferbezug im Sinne einer hohen Handlungsorientierung und einer Übertragung des Gelernten in die Praxis (SK4, SK5) laden auf dem konstruktivistischen Faktor. Je- 
doch weisen sie sowohl in der EFA als auch beim ESEM-Verfahren eher niedrige Faktorladungen auf. Es ist zu vermuten, dass transferförderliche Strategien Ausdruck eines konstruktivistischen Lehransatzes sind, jedoch ein konstruktivistischer Lehransatz noch nicht bedeutet, dass transferförderliche Strategien ebenso wie andere konstruktivistische Strategien verwendet werden. Auch dieser Aspekt sollte im Rahmen zukünftiger Studien gesondert untersucht werden. Die niedrigen Faktorladungen stehen vermutlich auch im Zusammenhang mit dem niedrigen $N$ der beiden Stichproben, die mit ca. 200 im gerade noch akzeptablen Bereich liegen (vgl. Bühner 2011).

Die Überprüfung der Skalenstruktur mit dem ESEM-Verfahren wies zunächst keinen akzeptablen Modell-Fit auf. Erst nach der Spezifikation von drei Korrelationen zwischen Items auf Basis der Modification Indices, zeigte das Modell einen akzeptablen Fit. Auch wenn Modellspezifikationen dieser Art nur mit Vorsicht zu verwenden sind (Wang und Wang 2012), können sie in diesem Fall gut durch die inhaltliche Ähnlichkeit der Items begründet werden.

Die Itemkennwerte der Skala LA-T zeigen gute Trennschärfen und Itemschwierigkeiten. Die Skala LA-K zeigt hingegen etwas niedrigere Trennschärfen und Itemschwierigkeiten. Bereits in der qualitativen Vorstudie hat sich gezeigt, dass ein konstruktivistischer Lehransatz insbesondere in Bezug auf die Strategien ein sehr heterogenes Konstrukt ist. Die niedrigen Trennschärfen sprechen unseres Erachtens dafür, dass die konstruktivistischen Strategieitems kein homogenes Konstrukt abbilden, sondern unterschiedliche Facetten konstruktivistischer Strategien. Bezüglich der niedrigen Itemschwierigkeit der Skala LA-K ist zu vermuten, dass Lehrende in der Weiterbildung insgesamt eher konstruktivistisch orientiert sind. Es könnte auch sein, dass es einen sozialen Erwünschtheitseffekt für hohe Werte auf der Skala LA-K bzw. umgekehrt für niedrige Werte auf der LA-T Skala gibt. Dies müsste in einer weiteren Studie unter Einbezug externer Ratings durch Teilnehmende und externe Beobachter überprüft werden.

Beide Subskalen zeigen eine gute interne Konsistenz. Die Hypothesen zur Skalenstruktur konnten jeweils angenommen werden. Die erwartete negative Skaleninterkorrelation entspricht den Ergebnissen bisheriger Studien (z. B. Braun und Hannover 2008; Lübeck 2010; Trigwell und Prosser 2004) und bestätigt damit ebenfalls die Zweidimensionalität von Lehransätzen. Die Korrelationen mit den verwendeten Methoden fallen jeweils für die Skalen LA-T und LA-K hypothesenkonform aus. Auch wenn es für eine vollständige Überprüfung der Validität noch weiterer Untersuchungen in zukünftigen Projekten bedarf, sind die erwartungskonformen Zusammenhänge erste Hinweise für die Validität der Subskalen.

Da die Zielgruppe für die Anwendung der Skala als auch der Weiterbildungskontext an sich sehr heterogen sind, wäre in zukünftigen Studien die Messmodellinvarianz zu prüfen, z.B. in Bezug auf die faktorielle Stabilität bei unterschiedlichen Weiterbildungsmerkmalen (z. B. Dauer oder thematischer Schwerpunkt) oder unterschiedlichen Merkmalen von Lehrenden (z. B. Qualifikationshintergrund oder Berufserfahrung). Aufgrund der Stichprobengröße war eine solche Prüfung im Rahmen unserer Studie nicht möglich.

Insgesamt zeigt sich damit die Lehransatz-Skala als ein Instrument, das erstmals eine reliable und ökonomische Erfassung von transmissiven und konstruktivistischen 
Lehransätzen von Lehrenden in der Weiterbildung ermöglicht. Erste Indikatoren für die Konstrukt- und Kriteriumsvalidität liegen ebenfalls vor. $\mathrm{Zu}$ beachten ist jedoch, dass die Lehransatz-Skala anhand einer Stichprobe von Lehrenden aus der beruflichen Weiterbildung entwickelt wurde. Es sind daher weitere Studien notwendig, um die Reliabilität und Validität der Skala auch für andere Weiterbildungsbereiche zu überprüfen. Darüber hinaus stellt die Lehransatz-Skala ein Instrument dar, das auf Selbstauskunft basiert und damit einem subjektiven Bias unterliegt (vgl. Podsakoff et al. 2012). In zukünftigen Studien wäre daher z. B. unter Hinzunahme externer Ratings von Weiterbildungsteilnehmenden oder durch externe Beobachter zu prüfen, inwiefern die Lehransätze auch mit dem tatsächlich beobachtbaren Lehrhandeln zusammenhängen. Hier ließe sich jedoch nur der Aspekt der Strategien erfassen, nicht der der Intentionen.

Die bisherige Forschung hat gezeigt, dass Lehransätze ein wichtiger Teil professioneller Lehrkompetenz sind. Unser Messinstrument stellt eine ökonomische und dem Kontext entsprechende Möglichkeit zur Messung von Lehransätzen von Lehrenden in der Weiterbildung dar. Es legt die Basis für die weitere Erforschung der Bedeutung unterschiedlicher Ausprägungen von Lehransätzen für den Weiterbildungserfolg und ermöglicht somit die weitere Erforschung der professionellen Lehrkompetenz von Lehrenden in der Weiterbildung.

Funding Open Access funding enabled and organized by Projekt DEAL.

Open Access Dieser Artikel wird unter der Creative Commons Namensnennung 4.0 International Lizenz veröffentlicht, welche die Nutzung, Vervielfältigung, Bearbeitung, Verbreitung und Wiedergabe in jeglichem Medium und Format erlaubt, sofern Sie den/die ursprünglichen Autor(en) und die Quelle ordnungsgemäß nennen, einen Link zur Creative Commons Lizenz beifügen und angeben, ob Änderungen vorgenommen wurden.

Die in diesem Artikel enthaltenen Bilder und sonstiges Drittmaterial unterliegen ebenfalls der genannten Creative Commons Lizenz, sofern sich aus der Abbildungslegende nichts anderes ergibt. Sofern das betreffende Material nicht unter der genannten Creative Commons Lizenz steht und die betreffende Handlung nicht nach gesetzlichen Vorschriften erlaubt ist, ist für die oben aufgeführten Weiterverwendungen des Materials die Einwilligung des jeweiligen Rechteinhabers einzuholen.

Weitere Details zur Lizenz entnehmen Sie bitte der Lizenzinformation auf http://creativecommons.org/ licenses/by/4.0/deed.de. 


\section{Anhang}

\section{Lehransatz-Skala}

\section{Strategien}

Tab. 6 Geben Sie im Folgenden bitte an, in welchem Maße Sie die einzelnen Lehrhandlungen im Rahmen der von Ihnen ausgewählten Veranstaltung verwendet haben. $1=$ nie, $6=\mathrm{in}$ sehr hohem Maße

\begin{tabular}{ll}
\hline ST1 & Ich bin aktiv gewesen und die Teilnehmenden haben zugehört \\
ST2 & Ich habe den Teilnehmenden Inhalte präsentiert \\
ST3 & Ich habe Inhalte durch Vorträge oder Präsentationen vermittelt \\
ST4 & Der Schwerpunkt der Veranstaltung lag auf der Vermittlung von Wissen und Informationen \\
SK1 & Ich habe flexibel auf den Bedarf der Teilnehmenden reagiert \\
SK2 & Die Teilnehmenden konnten über die Inhalte miteinander diskutieren \\
SK3 & Die Teilnehmenden haben die Möglichkeit zur Selbstreflexion erhalten \\
SK4 & $\begin{array}{l}\text { Die Teilnehmenden haben selbständig Lösungsvorschläge für Aufgaben oder Problemstel- } \\
\text { lungen erarbeitet }\end{array}$ \\
SK5 & $\begin{array}{l}\text { Ich habe den Teilnehmenden die Möglichkeit gegeben, das Gelernte in die Praxis zu über- } \\
\text { tragen }\end{array}$ \\
\hline
\end{tabular}

\section{Intentionen}

Tab. 7 Bitte geben Sie im Folgenden an, wie sehr die folgenden Aussagen auf die von Ihnen gewählte Veranstaltung zutreffen. 1 = trifft überhaupt nicht $\mathrm{zu}, 6=$ trifft voll und ganz zu

\begin{tabular}{ll}
\hline IT1 & Die Teilnehmenden sollten Wissen/Informationen vermittelt bekommen \\
IT2 & $\begin{array}{l}\text { Die Teilnehmenden sollten fachliche Inhalte vermittelt bekommen, um eine Wissensbasis } \\
\text { zu diesem Thema aufzubauen }\end{array}$ \\
IT3 & Die Teilnehmenden sollten Daten und Fakten zum Thema vermittelt bekommen \\
IT4 & Die Teilnehmenden sollten Inhalte lernen, die auch in (Fach-)Büchern zum Thema stehen \\
IK1 & Die Teilnehmenden sollten sich persönlich weiterentwickeln \\
IK2 & Die Teilnehmenden sollten ihre Sicht auf das Thema hinterfragen \\
IK3 & Die Teilnehmenden sollten Veränderungsimpulse bekommen \\
IK4 & Ich wollte, dass die Veranstaltung Transfer ermöglicht \\
\hline
\end{tabular}

\section{Literatur}

Aguinis, H., \& Kraiger, K. (2009). Benefits of training and development for individuals and teams, organizations, and society. Annual Review of Psychology, 60, 451-474.

Asparouhov, T., \& Muthén, B. (2009). Exploratory structural equation modeling. Structural Equation Modeling: A Multidisciplinary Journal, 16(3), 397-438.

Baldwin, T. T., \& Ford, J.K. (1988). Transfer of training: a review and directions for future research. Personnel Psychology, 41(1), 63-105.

Baldwin, T. T., Ford, J. K., \& Blume, B. D. (2017). The state of transfer of training research: moving toward more consumer-centric inquiry. Human Resource Development Quarterly, 28(1), 17-28. 
Baumert, J., \& Kunter, M. (2006). Stichwort: Professionelle Kompetenz von Lehrkräften. Zeitschrift für Erziehungswissenschaft, 9, 469-520.

Beausaert, S. A., Segers, M.S. R., \& Wiltink, D.P. (2013). The influence of teachers' teaching approaches on students' learning approaches: the student perspective. Educational Research, 55(1), 1-15.

Blume, B. D., Ford, J. K., Baldwin, T. T., \& Huang, J. L. (2010). Transfer of training: a meta-analytic review. Journal of Management, 36(4), 1065-1105.

Bonnes, C., \& Hochholdinger, S. (2020). Approaches to teaching in professional training: a qualitative study. Vocations and Learning, 13(3), 459-477.

Braun, E., \& Hannover, B. (2008). Zum Zusammenhang von Lehr-Orientierung und Lehrgestaltung von Hochschuldozierenden und subjektivem Kompetenzzuwachs bei Studierenden. In M. A. Meyer, M. Prenzel \& S. Hellekamps (Hrsg.), Perspektiven der Didaktik (Zeitschrift für Erziehungswissenschaft: Sonderheft 9, S. 277-291. https://doi.org/10.1007/978-3-531-91775-7_18). Wiesbaden: Springer VS.

Bühner, M. (2011). Einführung in die Test- und Fragebogenkonstruktion. München: Pearson Studium.

Burke, L. A., \& Hutchins, H. M. (2007). Training transfer: an integrative literature review. Human Resource Development Review, 6(3), 263-296.

Cohen, J. (1960). A coefficient of agreement for nominal scales. Educational and Psychological Measurement, 10(1), 37-46.

Dunn, T. J., Baguley, T., \& Brunsden, V. (2014). From alpha to omega: a practical solution to the pervasive problem of internal consistency estimation. British Journal of Psychology, 105(3), 399-412.

Fendler, J., \& Gläser-Zikuda, M. (2013). Teaching experience and the "shift from teaching to learning". Zeitschrift für Hochschulentwicklung, 8(3), 15-28.

Harshman, J., \& Stains, M. (2017). A review and evaluation of the internal structure and consistency of the Approaches to Teaching Inventory. International Journal of Science Education, 39(7), 918-936.

Hochholdinger, S., \& Keller, I. (2015). Welche Zusammenhänge bestehen zwischen den affektiven Reaktionen von Weiterbildungsteilnehmenden, der Lehrorientierung von Weiterbildungspersonal und Weiterbildungsinhalten? Zeitschrift für Erziehungswissenschaften, 18(1), 123-144.

Hochholdinger, S., \& Leidig, I. (2012). Wie lässt sich die professionelle Handlungskompetenz von Trainern/Trainerinnen ermitteln? In G. Niedermair (Hrsg.), Evaluation als Herausforderung der Berufsbildung und Personalentwicklung (S. 351-368). Linz: Trauner.

Hu, L. T., \& Bentler, P. M. (1999). Cutoff criteria for fit indexes in covariance structure analysis: conventional criteria versus new alternatives. Structural equation modeling: a multidisciplinary journal, 6(1), 1-55.

Kelava, A., \& Moosbrugger, H. (2012). Deskriptivstatistische Evaluation von Items (Itemanalyse) und Testwertverteilungen. In H. Moosbrugger \& A. Kelava (Hrsg.), Testtheorie und Fragebogenkonstruktion (S. 75-102). Heidelberg: Springer.

Kember, D. (1997). A reconceptualisation of the research into university academics' conceptions of teaching. Learning and Instruction, 7(3), 255-275.

Kline, R. B. (2016). Principles and practice of structural equation modeling. New York: Guildford.

König, J., \& Blömeke, S. (2009). Pädagogisches Wissen von angehenden Lehrkräften: Erfassung und Struktur von Ergebnissen der fachübergreifenden Lehrerausbildung. Zeitschrift für Erziehungswissenschaft, 12(3), 499-527.

Lindblom-Ylänne, S., Trigwell, K., Nevgi, A., \& Ashwin, P. (2006). How approaches to teaching are affected by discipline and teaching context. Studies in Higher Education, 31(3), 285-298.

Little, T. D. (2013). Longitudinal structural equation modeling. New York: Guilford.

Lübeck, D. (2010). Wird fachspezifisch unterschiedlich gelehrt? Empirische Befunde zu hochschulischen Lehransätzen in verschiedenen Fachdisziplinen. Zeitschrift für Hochschulentwicklung, 5, 7-24.

Mak, B., \& Chik, P. (2011). Differences in perceived approaches to learning and teaching English in Hong Kong secondary schools. Educational Review, 63(2), 195-218.

Marsh, H.W., Lüdtke, O., Muthén, B., Asparouhov, T., Morin, A.J., Trautwein, U., \& Nagengast, B. (2010). A new look at the big five factor structure through exploratory structural equation modeling. Psychological Assessment, 22(3), 471.

Marsh, H.W., Morin, A.J., Parker, P.D., \& Kaur, G. (2014). Exploratory structural equation modeling: an integration of the best features of exploratory and confirmatory factor analysis. Annual Review of Clinical Psychology, 10, 85-110.

Mayring, P. (2015). Qualitative Inhaltsanalyse. Grundlagen und Techniken. Weinheim: Psychologie Verlags Union.

Meyer, J.H., \& Eley, M.G. (2006). The approaches to teaching inventory: a critique of its development and applicability. British Journal of Educational Psychology, 76(3), 633-649. 
Peters, G.-J. (2018). Userfriendlyscience: quantitative analysis made accessible. R package version 0.7.2. https://doi.org/10.17605/osf.io/txequ.

Podsakoff, P. M., MacKenzie, S.B., \& Podsakoff, N.P. (2012). Sources of method bias in social science research and recommendations on how to control it. Annual Review of Psychology, 63, 539-569.

Prosser, M., \& Trigwell, K. (2006). Confirmatory factor analysis of the approaches to teaching inventory. British Journal of Educational Psychology, 76(2), 405-419.

Rosário, P., Núñez, J.C., Vallejo, G., Paiva, O., Valle, A., Fuentes, S., \& Pinto, R. (2014). Are teachers' approaches to teaching responsive to individual student variation? A two-level structural equation modeling. European Journal of Psychology of Education, 29(4), 577-601.

Salas, E., Tannenbaum, S. I., Kraiger, K., \& Smith-Jentsch, K. A. (2012). The science of training and development in organizations: what matters in practice. Psychological Science in the Public Interest, $13,74-101$.

Schaper, N., \& Sonntag, K. (2007). Wissensorientierte Verfahren der Personalentwicklung. In H. Schuler \& K. Sonntag (Hrsg.), Handbuch der Arbeits- und Organisationspsychologie (S. 602-612). Göttingen: Hogrefe.

Schermelleh-Engel, K., Moosbrugger, H., \& Müller, H. (2003). Evaluating the fit of structural equation models: tests of significance and descriptive goodness-of fit measures. Methods of Psychological Research Online, 8, 23-74.

Schrader, J. (2010). Teilnehmerorientierung. In R. v. Arnold, S. Nolda \& E. Nuissl (Hrsg.), Wörterbuch Erwachsenenbildung (S. 284-285). Stuttgart: Julius Klinkhardt/UTB.

Seifried, J. (2009). Unterricht aus der Sicht von Handelslehrern. Frankfurt a. M.: Peter Lang.

Sonntag, K., \& Stegmaier, R. (2007). Verhaltensorientierte Verfahren der Personalentwicklung. In H. Schuler \& K. Sonntag (Hrsg.), Handbuch der Arbeits- und Organisationspsychologie (S. 613-624). Göttingen: Hogrefe.

Stes, A., \& Van Petegem, P. (2014). Profiling approaches to teaching in higher education: a cluster-analytic study. Studies in Higher Education, 39(4), 644-658.

Trigwell, K., \& Prosser, M. (2004). Development and use of the approaches to teaching inventory. Educational Psychology Review, 16(4), 409-424.

Trigwell, K., Prosser, M., \& Taylor, P. (1994). Qualitative differences in approaches to teaching first year university science. Higher Education, 27, 75-84.

Trigwell, K., Prosser, M., \& Waterhouse, F. (1999). Relations between teachers' approaches to teaching and students' approaches to learning. Higher Education, 37(1), 57-70.

Velicer, W.F., Eaton, C. A., \& Fava, J.L. (2000). Construct explication through factor or component analysis: a review and evaluation of alternative procedures for determining the number of factors or components. In R.D. Goffin \& E. Helmes (Hrsg.), Problems and solutions in human assessment (S. 41-71). Boston: Kluwer.

Wang, J., \& Wang, X. (2012). Structural equation modeling: applications using Mplus. Chichester: Wiley. 